\title{
Multimodal Biometrics for Improving Automatic Teller Machine Security
}

\author{
S. Pravinthraja and Dr.K. Umamaheswari
}

\begin{abstract}
A multimodal biometrics face and fingerprint recognition system is a computer application for automatically identifying or verifying a person from face by using cameras and fingerprint by using sensors or fingerprint readers or fingerprint scanners. Proposed paper uses Face and Fingerprint recognition technique for verification in ATM systems. There are two types. The first one is verification. Compare the two faces and fingerprint images and decide whether the user (current user image) is an genuine user or imposter. These are decision level. Second one is identification this is where the system compares the given input image to all other images in the database and gives a ranked list of matches. Multimodal biometrics verification system that verifies the presence of a user is genuine or not. Two modalities are currently used-face and fingerprint-but our theory can be readily extended to include more modalities. We show that verification imposes additional requirements on multimodal fusion when compared to conventional verification systems. We also argue that the usual performance metrics of false accept and false reject rates are insufficient yardsticks for continuous verification and propose new metrics against which we benchmark our system.
\end{abstract}

Keywords--- Pattern, Face Recognition, Fingerprint Recognition, Identification, Verification

\section{INTRODUCTION}

$\mathrm{I}$ $\mathrm{N}$ this paper, we present an implementation of a security system based on Face and Fingerprint. Verification algorithm is developed using The information age is quickly revolutionizing the way transactions are completed. Everyday actions are increasingly being handled electronically, instead of with pencil and paper or face to face. This growth in electronic transactions has resulted in a greater demand for fast and accurate user identification and authentication. Access codes for buildings, banks accounts and computer systems often use PIN's for identification and security clearances. Using the proper PIN gains access, the successful transactions can occur, but the user of the PIN is not verified. When ATM cards are lost or stolen, an unauthorized user can often come up with the correct personal codes. This paper describes how face and fingerprint recognition technology can help to the real world ATM machines. Face recognition technology

S. Pravinthraja, Research Scholar, AP, Department of CSE, Sri Ramakrishna Institute of Technology, E-mail: pravinthraja@gmail.com

Dr.K. Umamaheswari, Professor, Department of Information Technology, PSG College of Technology, Coimbatore, Tamilnadu, India, Email: umakpg@gmail.com analyzes the unique shape, pattern and positioning of the facial features. Face recognition is very complex technology and is largely software based. This Biometric Methodology establishes the analysis framework with PCA algorithms for each type of biometric device. The analysis of fingerprints for matching purposes generally requires the comparison of several features of the fingerprint patterns, which are aggregate characteristics of ridges, and minutia points, which are unique features found within the patterns. With the help of these features we can identify the genuine user from different imposters. In this paper, we present a holistic fusion method that combines face and fingerprint across modalities and time simultaneously. This is realized by using a Hidden Markov Model in a Bayesian framework. Our method lends itself to an efficient recursive formulation and requires only one usersupplied parameter. This parameter determines how quickly old observations are discounted and can be tuned by the security administrator to fit the security policy of the site.

\section{EXISTING SYSTEM}

Previously, the most popular methods of keeping information and Accessing money from ATM centers are used ATM cards and UserID/PIN protection. These schemes require the users to authenticate themselves by entering a "secret "password that they had previously created or were assigned. When ATM cards are lost or stolen, these systems are prone to hacking, either from an attempt to crack the password or from passwords which were not unique. A Biometric Identification system is one in which the user's "body "becomes the password/PIN. Biometric characteristics of an individual are unique and therefore can be used to authenticate a user's access to ATM centers.

Previous methods of authentication in ATM centers are Unimodal Biometric methodologies [8].These Unimodal identification and verification systems are using single Biometric characteristic. In this paper presents a new methodologies adding one more characteristic multimodal biometric characteristic (face and fingerprint) for authentication purpose. Proposed methodology of multimodal biometric characteristic secured accessing in ATM Centers.

\section{PROPOSED SYSTEM}

\subsection{Face Recognition System}

Figure 1 below shows the typical way that a facial recognition system can be made operational. 


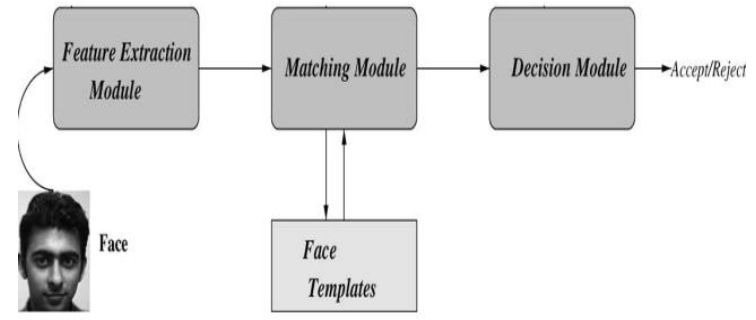

Figure 1: Overview of FRS

The first step is the capturing of a face image. This would normally be done using a still or video camera. The face image is passed to the recognition software for recognition (identification or verification). This would normally involve a number of steps such as normalizing the face image and then creating a 'template' of 'print' to be compared to those in the database. The match can either be a true match which would lead to investigative action or it might be a 'false positive 'which means the recognition algorithm made a mistake and the alarm would be cancelled. Each element of the system can be located at different locations within a network, making it easy for a single operator to respond to a variety of systems.

\subsubsection{Principal Component Analysis}

Principal component analysis (PCA) involves a mathematical procedure which extracts facial features for recognition, this approach transforms face images into a small set of characteristic feature images called eigenfaces. The first principal component accounts for as much of the variability in the data as possible, and each succeeding component accounts for as much of the remaining variability as possible.

These methods capture the local facial features and their geometric relationships. They often locate anchor points at key facial features (eyes, nose, mouth, etc), connect these points to form a net and then measure the distances and angles of the net to create a unique face 'print'.

\subsection{Fingerprint Recognition System}

Figure 2 below shows the typical way that a fingerprint recognition system can be made operational.

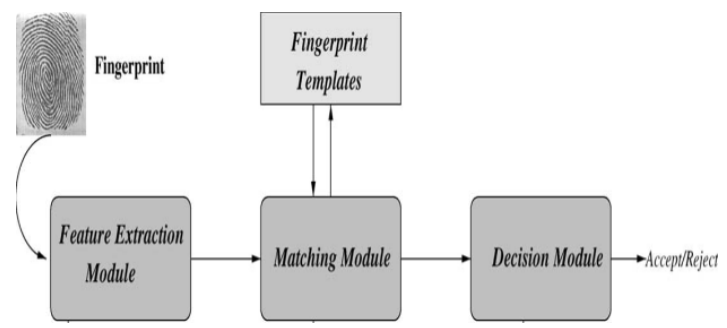

Figure 2: Overview of FPRS

The first step is the capturing of a fingerprint image. This would normally be done using a fingerprint scanner or Reader. The fingerprint image is passed to the recognition software for recognition number of steps such as normalizing the fingerprint image and then creating a 'template' of 'print' to be compared to those in the database. The match can either be a true match which would lead to investigative action or it might be a 'false positive which means the recognition algorithm made a mistake and the alarm would be cancelled. Each element of the system can be located at different locations within a network, making it easy for a single operator to respond to a variety of systems.

\subsubsection{Fingerprint Verification}

This paper introduces a prototype automatic identity authentication system that is capable of authenticating the identity of an individual, using fingerprints. The main

1. Components of the AFIS are

2. Fingerprint database

3. Fingerprint features database

4. Enrollment Module

5. Authentication module.

The fingerprint database stores the fingerprint images. For this purpose. The features extracted from these fingerprints are stored in the features database along with the person ID. The objective of the enrollment module is to admit a person using his/her ID and fingerprints into a fingerprints database after the process of feature extraction. These features form a template that is used to determine or verify the identity of the subject, formulating the process of authentication. The component of the AFIS used for authentication is referred to as the authentication module. Figure 3 illustrates the different steps involved in the development of the AFIS. The details of these steps are given in the following subsections.

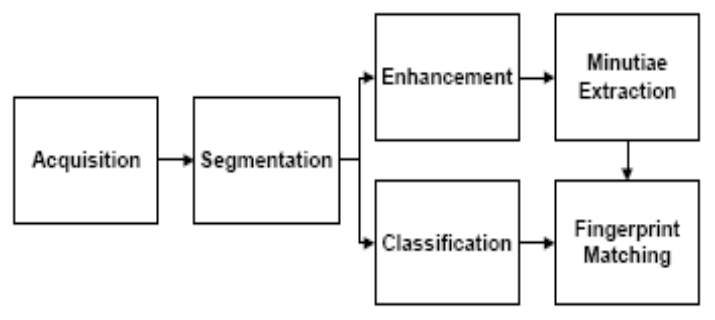

Figure 3: Overview of AFIS

\subsubsection{Fingerprint Segmentation}

Fingerprint segmentation is an important part of a fingerprint identification and verification system. This algorithm is based only on the block coherence of an image. Coherence gives us a measure of how well the gradients of the fingerprint image are pointing in the same direction. In a window of size $\mathrm{WxW}$ around a pixel, the coherence is defined as $\mathrm{Mc}$ is the global mean of the coherence image and Sc is its global standard deviation. Holes in the segmentation mask are removed using morphological post processing. This mask on pixel-wise multiplication with the fingerprint image gives the segmented image.

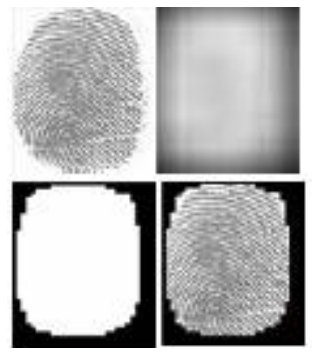

Figure 4: Segmented Image

A. Original Image, B. Coherence Image, C. Segmentation Mask, D) Segmented 


\subsection{Fingerprint Enhancement}

The fingerprint enhancement algorithm mentioned in [20] was found to be suitable for this application and was therefore used in the system. Better results are obtained using [22] but it is slightly more time consuming. This algorithm calls for the development of a ridge frequency image IRF and ridge orientation IRO image for a fingerprint. Gabor filters are used to enhance the fingerprint utilizing the ridge frequency and ridge orientation information

Obtained from the frequency and orientation images obtained earlier the enhanced image IE is then binarized using adaptive thresholding to give a binarized image IEB.

The binarized image is thinned to give IT. The thinned version is used for minutiae extraction.(see figure 6)

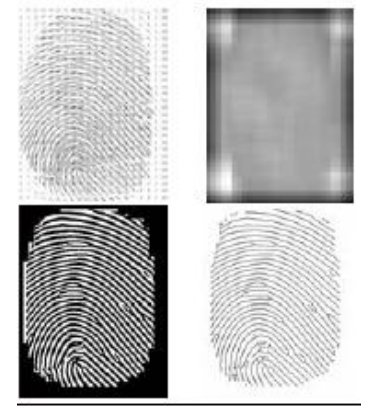

Figure 5: Minutiae Extraction

\section{A. Orientation Field, B. Ridge Frequency Image C. Enhanced Image D. Thinned Image}

\subsubsection{Minutia Features}

The major Minutia features of fingerprint ridges are: ridge ending, bifurcation, and short ridge (or dot). The ridge ending is the point at which a ridge terminates. Bifurcations are points at which a single ridge splits into two ridges. Short ridges (or dots) are ridges which are significantly shorter than the average ridge length on the fingerprint. Minutiae and patterns are very important in the analysis of fingerprints since no two fingers have been shown to be identical.

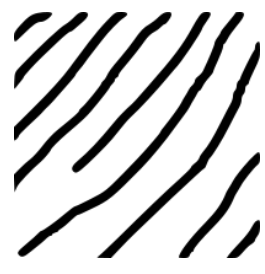

Ridge ending

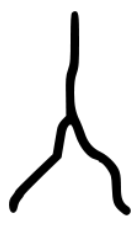

Bifurcation

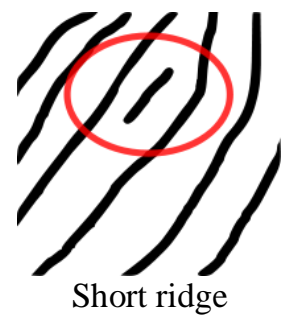

Figure 6: FP Images

\subsubsection{Minutiae Extraction}

Minutiae extraction was carried out using the crossing number approach. Crossing number of pixel ' $p$ ' is defined as half the sum of the differences between pairs of adjacent pixels defining the 8-neighborhood of ' $p$ '. Mathematically

$$
c n(p)=\frac{1}{2} \sum_{i=1.8}\left|\operatorname{val}\left(p_{i \bmod 8}\right)-\operatorname{val}\left(p_{i-1}\right)\right|
$$

Where $p 0$ to $p 7$ are the pixels belonging to an ordered sequence of pixels defining the 8-neighborhood of $p$ and $v a l$ $(p)$ is the pixel value
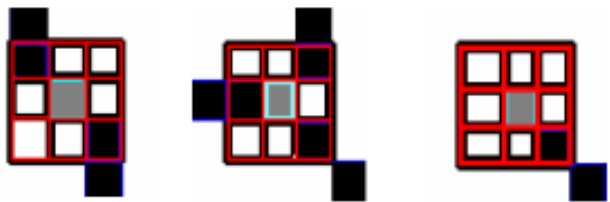

Figure 7: Non Minutiae Region

cn $(p)=2, c n(p)=3$ and $c n(p)=1$ representing a non minutiae region, a bifurcation and a ridge ending Crossing numbers 1 and 3 correspond to ridge endings and ridge bifurcations respectively. An intermediate ridge point has a crossing number of 2 . The minutiae obtained from this algorithm must be filtered to preserve only the true minutiae. The different types of false minutiae introduced during minutiae extraction include spike, bridge, hole, break, Spur, Ladder, and Misclassified Border areas. (See figure 8)

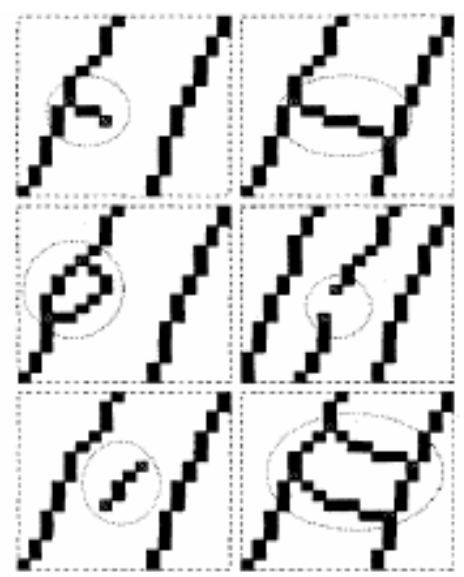

Figure 8: Different Types of False Minutiae

A. Spike, B. Bridge, C. Hole, D.Break, E. Spur F. Ladder

\subsubsection{Minutiae Matching}

Let $\mathrm{T}$ and $\mathrm{I}$ be the representation of the template and input fingerprint, respectively. Let the minutiae sets of the two Fingerprints be given by: A minutia mj' in I and a minutia mi in $\mathrm{T}$ are considered to be matched if their spatial and orientation differences are within specified thresholds ro and $\theta$ o.

$$
\begin{array}{ll}
\mathrm{T}=\left\{\mathrm{m}_{1}, \mathrm{~m}_{2}, \ldots \mathrm{m}_{\mathrm{n}}\right\} & \mathrm{m}_{\mathrm{i}}=\left\{\mathrm{x}_{\mathrm{i}}, \mathrm{y}_{\mathrm{i}}, \mathrm{\theta}_{\mathrm{i}}\right\}=1 . \mathrm{m} \\
\mathrm{I}=\left\{\mathrm{m}_{1}, \mathrm{~m}_{2}, \ldots \mathrm{m}_{\mathrm{n}}\right\} & \mathrm{m}_{\mathrm{j}}=\left\{\mathrm{x}_{\mathrm{j}}, \mathrm{y}_{\mathrm{j}}, \mathrm{\theta}_{\mathrm{j}}\right\}=1 . \mathrm{n}
\end{array}
$$

In this approach the minutiae sets are first registered using a derivative of the Hough transform, followed by fingerprint 
matching using spatial and orientation-based distance computation. The matching algorithm returns a percentage match score, which is then used to take the match-no match decision based on the security criterion.

\section{HOLISTIC FUSION}

At the heart of our technique is the integration of biometric observations across modalities and over time. This is done by the Integrator using an HMM. which is a sequence of states $\mathrm{xt}$ that "emit" observations zt, for time $t 1 / 41 ; 2 ; \ldots$ Each state can assume one of two values: xt $2 \mathrm{~S}$, where $\mathrm{S} 1 / 4 \mathrm{f}$ Safe; Attacked

The state Safe means that the logged-in user is still present at the computer console, while the state Attacked means that an imposter has taken over control. It is also possible for the user to be absent from the console, but, for a high security environment, this is considered to be the same as Attacked. Each observation ztis either a face or fingerprint image or, equivalently, its corresponding score. The result of the fusion is the calculation of Psafe, the probability that the system is still in the Safe state. This value can then be compared to a predefined threshold Tsafe set by the security administrator, below which appropriate action may be taken. A key feature of our method is that we can compute Psafe at any point in time whether or not there are biometric observations .In the absence of observations, there is a built-in mechanism to decay Psafe reflecting the increasing uncertainty that the system is still safe.

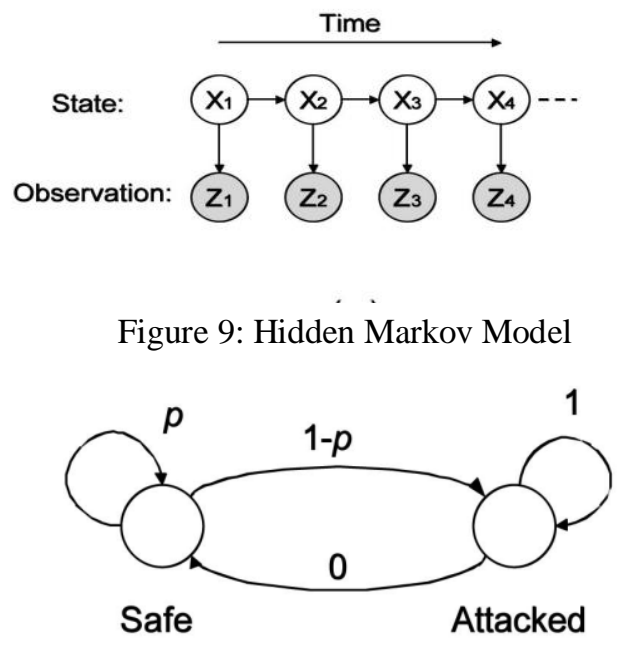

Figure 10: State Transition Diagram

\section{FUSION IN BIOMETRICS}

The layout of a bimodal system is shown in Fig.11. The purpose of this diagram is to illustrate the various levels of fusion for combining two (or more) biometric systems. The three possible levels of fusion are: (a) fusion at the feature extraction level, (b) fusion at the matching score level, (c) fusion at the decision level.

(1) Fusion at the feature extraction level: The data obtained from each sensor is used to compute a feature vector. As the features extracted from one biometric trait are independent of those extracted from the other, it is reasonable to concatenate the two vectors into a single new vector. The new feature vector now has a higher Dimensionality and represents a persons identity in a different (and hopefully more discriminating) hyperspace. Feature reduction techniques may be employed to extract useful features from the larger set of features.

(2) Fusion at the matching scores level: Each system provides a matching score indicating the proximity of the feature vector with the template vector. These scores can be combined to assert the veracity of the claimed identity Techniques such as logistic regression may be used to combine the scores reported by the two sensors. These techniques attempt to minimize the FRR for a given FAR (Jain et al., 1999b).

(3) Fusion at the decision level: Each sensor can capture multiple biometric data and the resulting feature vectors individually classified into the two classes-accept or reject. A majority vote scheme, such as that employed in (Zuev and Ivanon, 1996) can be used to make the final decision.

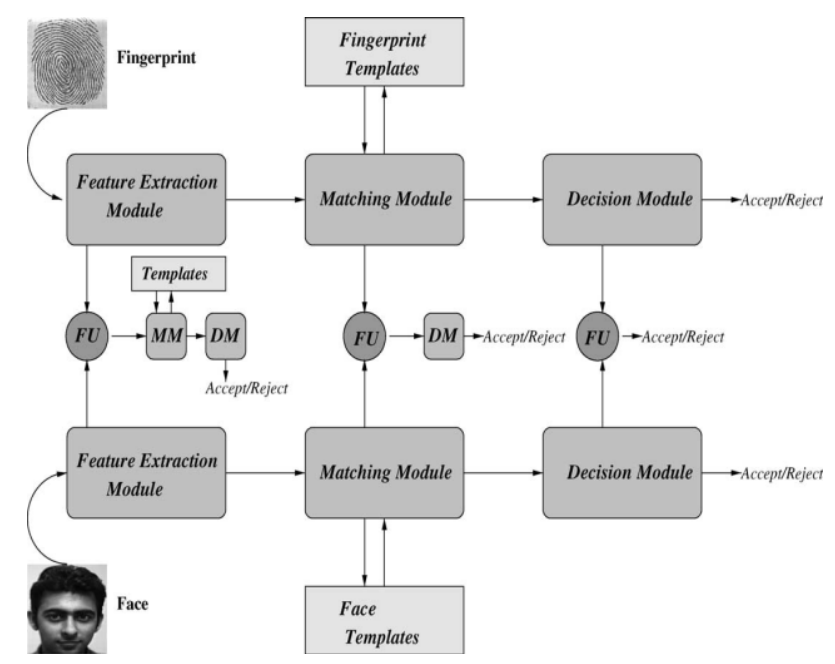

Figure11: Three Levels of Fusion

(FU: Fusion Module, MM: Matching Module, DM: Decision Module)

\section{MultiPle Biometric Fusion}

Multibiometric fusion refers to the fusion of multiple biometric indicators. Such systems seek to improve the speed and reliability (accuracy) of a biometric system (Rattani, A.; Kisku, 2007) by integrating matching scores obtained from multiple biometric sources. A variety of fusion schemes have been described in the literature to combine these various scores. These include majority voting, sum and product rules, k-NN classifiers, SVMs, decision trees, Bayesian methods, etc. .An important aspect that has to be addressed in fusion at the matching score level is the normalization of the scores obtained from the different domain experts .Normalization typically involves mapping the scores obtained from multiple domains into a common domain before combining them. This could be viewed as a two-step process in which the 
Distributions of scores for each domain is first estimated using robust statistical techniques and these distributions are then scaled or translated into a common domain. Besides the techniques described above, other types of fusion are also possible in biometrics:

(i) A Fingerprint biometric system may store multiple templates of a users fingerprint (same finger) in its database. When a fingerprint impression is presented to the system for verification, it is compared against each of the templates, and the matching score generated by these multiple matching's are integrated.

(ii) A system may store a single template of a user's finger, but acquire multiple impressions of the finger during verification.

(iii) Another possibility would be to acquire and use impressions of multiple fingers for every user. These possibilities have been discussed in (Jain et al., 1999e).

\section{EXPERIMENTAL RESULTS}

A brief description of the two biometric indicators used in our experiments is given below.

\subsection{Face Recognition Vendor Test}

The medium size database consisted of number outdoor images from various sources. Figure 12 below gives an indication of the images in the database.

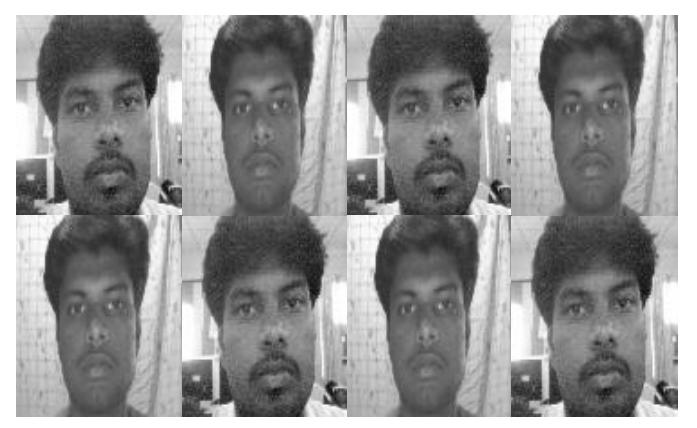

Figure 12: Various Poses Images from the Medium Data Base

With the very good images from the large database (37,437 images) the identification performance of the best system at rank one is $96 \%$ at a false accept rate of $1 \%$.

\section{A. The Size of the Database}

The Face Recognition Vendor Test (FRVT) has recognized the face recognition in four technical areas.They are high resolution still imagery, 3D facial scans,multi sample still facial imagery and preprocessing algorithm (PCA) that compensate pose and illumination.

\section{B. Individual's Face}

The method of defining the matrix varies according to the algorithm (the mathematical process used by the computer to perform the comparison).

Here the part inside the oval is chosen and the other parts are rejected, artificial intelligence is used to simulate human interpretation of faces.

In order to increase the accuracy and adaptability, some kind of machine learning of 3D face tracking, 3D face reconstruction has to be implemented.

\subsection{Face Database Samples}

Face database consist of two types there are

1. Test images

2. Trained Images

\subsubsection{Trained Image Samples}

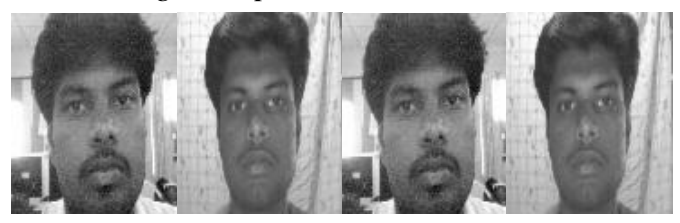

Figure 13: Various Trained Images from the Medium Data Base

These face images are authorised user images stored from the databases.

\subsubsection{Test Image Samples}

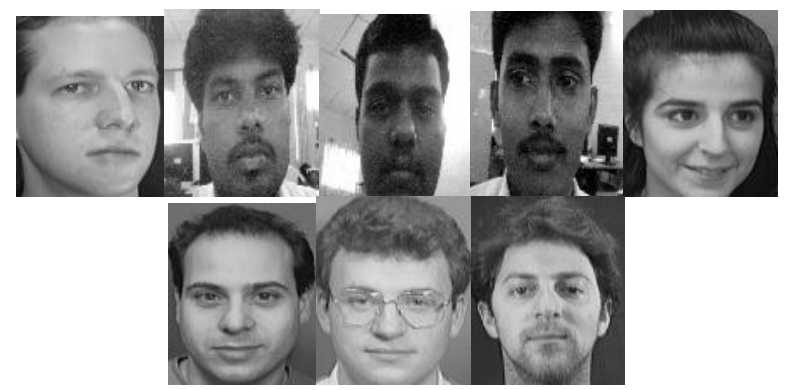

Figure 14: Various Test Images Captured from the Cameras

These different face images are compared with the trained images(original user images).Test images are captured from different users by face cameras.If these captured face images are matched with the original image available in the database then the particular user can be allowed to access the ATM machines (see below fig)otherwise the users are not allowed.

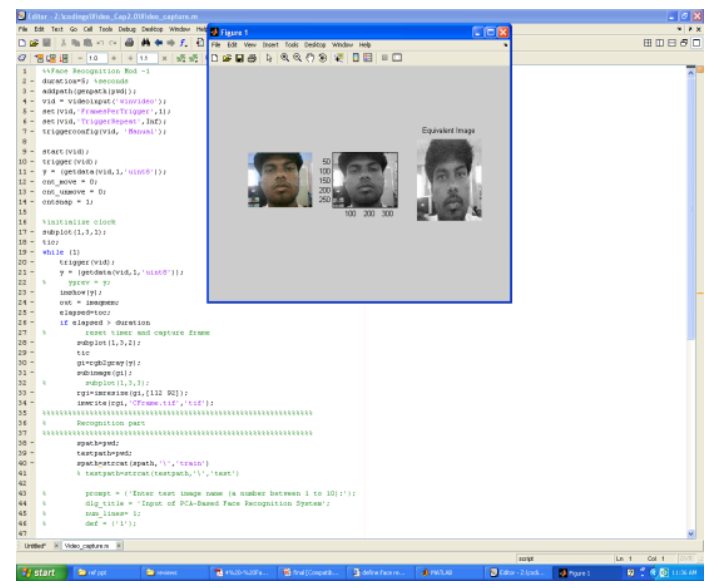

Figure 15: Captured Image is Compared with the Database

\subsection{Fingerprint Verification}

A fingerprint refers to the flowing pattern of Ridges and furrows located on the tip of a finger. Traditionally, these patterns have been extracted by creating an inked impression of the fingertip on paper. But the electronic era has ushered in 
a range of compact sensors that provide digital images of these patterns (Fig. 4). In real-time verification systems, the images acquired by these sensors are used by the feature extraction module to compute the feature values. The feature values typically correspond to the position and orientation of certain critical points known as minutiae points (ridge endings and ridge bifurcations) that are present in every fingerprint (Fig. 5). The matching process involves comparing the twodimensional minutiae patterns extracted from the users print with those in the template. For this experiment, the techniques described in (Jain et al., 1997) were used in the feature extraction and matching stages of the fingerprint verification system.

\subsubsection{Fingerprint Samples}

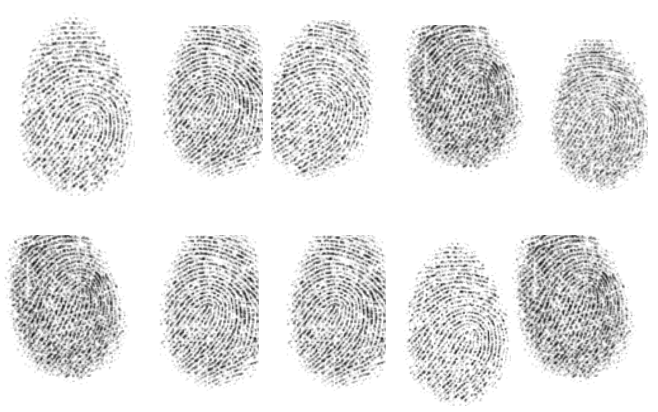

Figure 15: Various Fingerprint Images from the Medium Data Base

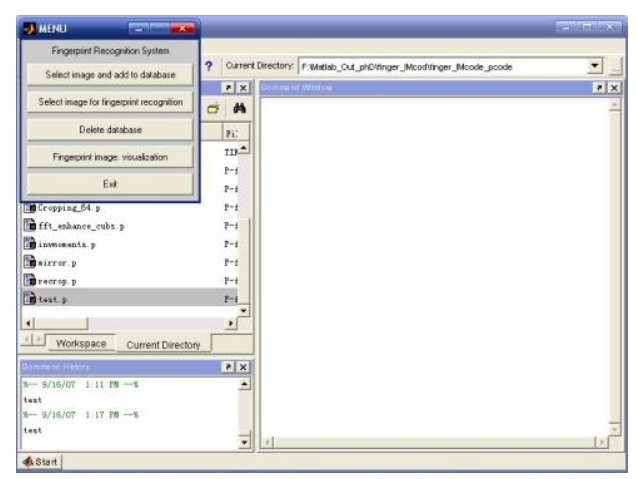

Figure 16: Fingerprint Verification by using MATLAB

The above figure shows fingerprint database and fingerprint verification and recognitoin using matlab.

\section{PERFORMANCE}

\section{A. False Acceptance Rate (FAR)}

The probability that a system will incorrectly identify an individual or will fail to reject an imposter. It is also called as type 2 error rate.

\section{$\mathrm{FAR}=\mathrm{NFA} / \mathrm{NIIA}$}

$\mathrm{NFA}=$ number of false acceptance

$\mathrm{NIIA}=$ number of imposter identification attempts.

\section{B. False Rejection Rate (FRR)}

The probability that a system will fail to identify an enrollee. It is also called type 1 error rate.

\section{$\mathrm{FRR}=\mathrm{NFR} / \mathrm{NEIA}$}

Where $\mathrm{FRR}=$ false rejection

$\mathrm{NFR}=$ number of false rejection rates

$\mathrm{NEIA}=$ number of enrollee identification attempt.

\section{Response Time}

The time period required by a biometric system to return a decision on identification of a sample.

\section{Threshold/ Decision Threshold}

The acceptance or rejection of a data is dependent on the match score falling above or below the threshold. The threshold is adjustable so that the system can be made more or less strict depending on the requirements of any given application.

\section{E. Enrollment Time}

The time period a person must spend to have his/her facial reference template successfully created.

\section{F. Equal Error Rate}

When the decision threshold of a system is set so that the proportion of false rejection will be approximately equal to the proportion of false acceptance.

\section{CONCLUSION}

This paper provides initial results obtained on a multimodal biometric system that uses face and fingerprint features for biometric Verification purposes. Our experiments indicate that better security than other methods like passwords, PIN numbers. The benefits of multibiometrics may become even more evident in the case of a larger database of users. Certain applications of recognition technologies are now cost effective, reliable and highly accurate.Our holistic fusion method to combine the face and fingerprint for high security purposes in ATM machines. Face and fingerprint recognition technology can be used worldwide to access buildings, however it can be used in ATMs, which would help address potential security threats in near future.

\section{REFERENCES}

[1] S. Kumar, T. Sim, R. Janakiraman, and S. Zhang, "Using Continous Biometrics Verification to Protect Interactive Login Sessions," Proc. 21st Ann. Computer Security Applications Conf.,pp. 441-450, 2005.

[2] S. Zhang, R. Janakiraman, T. Sim, and S. Kumar, "Continuous Verification Using Multimodal Biometrics," Proc. Second Int'l Conf. Biometrics, pp. 562-570, 2006.

[3] "Feature Level Fusion of Face and Fingerprint Biometrics"Rattani, A.; Kisku, D.R.; Bicego, M.; Tistarelli, M.; Univ. of Cagliari, Cagliari conf.,pp 27-29 Sept. 2007

[4] "Identity theft, computers and behavioral biometrics" Moskovitch, R.; Feher, C.; Messerman, A.; Kirschnick, N.; Mustafic, T.; Camtepe, A.; Lohlein, B.; Heister, U.; Moller, S.; Rokach, L.; Elovici, Y.; Deutsche Telekom Labs., Ben Gurion Univeristy, Beer-Sheva 8-11 June 2009

[5] "Biometrics Verification Techniques Combing with Digital Signature for Multimodal Biometrics Payment System"JuCheng Yang; Sch. of Inf. \& Technol., Jiangxi Univ. of Finance \& Econ., Nanchang, China 23-24 Oct. 2010

[6] "Combining feature level and matching score level fusion strategies for multi-biometrics "Ningbo $\mathrm{Zhu}$; $\mathrm{Fu} \mathrm{Yu}$; Qing long Tian; Hunan University, School of Computer and Communication, Changsha, China 
24-26 June 2011

[7] Security System Using Biometric Technology: Design and Implementation of Voice Recognition System (VRS) Rozeha A. Rashid, Nur Hija Mahalin, Mohd Adib Sarijari, Ahmad Aizuddin Abdul Azizmay-2008

[8] Improving atm security via face recognition K John Peter, G.Nagarajan, Dr S.Arguman, Dr. K Sentamarai Kannan conf.,pp2009

[9] A new framework for adaptive multimodal Biometrics management Ajay kumar, senior member, ieee, vivek kanhangad, student member, ieee, and david zhang, fellow, ieee 2011

[10] C. Vogler, Z. Li, A. Kanaujia, S. Goldenstein, and D. Metaxas. The best of both worlds: Combining $3 \mathrm{~d}$ de-formable models with active shape models. ICCV 2010. 